\title{
$\mathrm{Z}_{N}$ 模型的辫子群与量子群*
}

\author{
葛墨林赵宏康刘广群 \\ （南开数学研究所,天津 300071）（兰州大学物理系,兰州 730001）
}

\section{关链词 $\mathrm{Z}_{N}$ 模型、辫子群、量子群、Weyl 关系}

自从 Baxter 严格求解八顶角模型以来 ${ }^{[1]}$, 这个方向的研究引起了人们越来越大的兴趣. 这个模型可以约化为杨-Baxter 方程 (YBE) 的三角解, 进一步又可约化为有理解. 前者引出 了量子群 $S L_{q}(2)$, 后者则为 Yangian $Y(S L(2))^{[2]}$. 在 Sklyanin 工作中, 定义了 Sklyanin 代数 ${ }^{[3]}$, 这是与椭圆解相关的重要进展, 但从 Hopf 代数角度而言, 其 Coproduct 代数结构已 非通常量子代数的含义. 从另一个角度来研究这个问题, 则是从不依赖于谱参数的 $Z_{N}$-辫子 群表示 (BGR) 出发,然后再利用杨- Baxterization 方案 ${ }^{[4]}$, 给出 YBE 的解 ${ }^{[4,5]}$.

在我们以前工作中 ${ }^{[4,5]}$,一些具体的 BGR 形式可以构造出来,并在 $N-2$ 特殊情况下可 以证明它满足 free-fermion 条件,但是缺乏任意 $Z_{N}$ 时 BGR 的表达式, 同时也没有讨论其 量子群结构. 与 Tracy 一致 ${ }^{[6]}$, 我们定义与 $\mathrm{Z}_{N}$ 相联系的 BGR 为 $S$, 其矩阵形式满足

$$
\begin{aligned}
& S_{12} S_{23} S_{12}-S_{23} S_{12} S_{23}, \\
& S_{k i}^{i i}=0, \text { 除非 } i+j-k+l(\bmod N), \\
& S_{k+p, p+1+p}^{i+p}-S_{k i}^{i i}, \text { 对任意 } i, j, k, l, p \in Z_{N},
\end{aligned}
$$

其中 $S_{12}-S \otimes 1, S_{23}=1 \otimes S$. 在文献 $[3,4]$ 中我们讨论了满足(1)-(3)式的解, 现在我们将 把另一个新参数 $\omega\left(\omega^{N}=1\right)$ 引人这个解理. 以后我们将看到, 它对引起交换(非平凡)代数是 必要的. 为了说明这点, 首先讨论八顶点情况. 对 $Z_{2}$ 模型(八顶点)其一般形式为

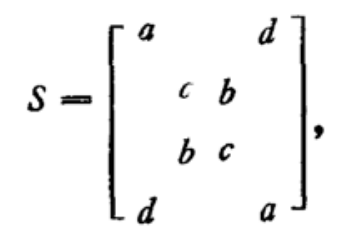

其中未标出的矩阵元为零. 将 (4) 式代人 (1)-(3) 式易知当且仅当 $a^{2}-b^{2}, c^{2}-d^{2}$ 时,才 得以满足. 于是, 利用标准的 $\mathrm{F}-\mathrm{R}-\mathrm{T}^{[7]}$ 技术, 便立即可求出其结合代数 $A(S)$, 其生成元设 为 $x_{i j} i, j=1,2$, 并满足

经过计算,可得到其表示

$$
S(x \otimes x)=(x \otimes x) S .
$$

$$
\pi(x)=\left[\begin{array}{cc}
a Z & \omega c Z X \\
c Z X & \omega a Z
\end{array}\right]
$$

其中 $Z$ 与 $X$ 为算子, $Z$ 满足 Weyl 关系

$$
Z X=\omega X Z, \omega^{2}=1 .
$$

有趣的是, 当取 $\omega=1$ 时, 所有生成元 $Z, X$ 均可对易,亦即在八顶角模型中, 生成元互易并 不一定对应 $S=I$. (7) 式的 $\omega$ 因子可视为一个新参数, 在本例中 $\omega^{2}-1$. 以下我们将这个 结果推向一般情况.

本文 1991 年 11 月 25 日收到.

* 国家自然科学基金资助项目. 


\section{一、 $\mathrm{Z}_{N}$ 禩 型}

可以证明,以下形式的 $S$ 满足 (1)一(3) 式所有要求:

$$
S_{k l}^{i j}-\sum_{0<n<N-1} w_{n} \delta^{(N)}(i-l-n) \delta^{(N)}(j-k+n) \omega^{i-k},
$$

其中

$$
\begin{aligned}
& \omega^{N}-1, \\
& \delta^{(N)}(m)=\left\{\begin{array}{l}
1, m=0(\bmod N), \\
0, \text { 当 } m \text { 为其他. }
\end{array}\right.
\end{aligned}
$$

事实上, 直接计算可证明 $\bar{S}$ 满足 (1)一(3) 式,因为 $\bar{S}$ 满足

$$
\bar{S}_{k l}^{\prime j}-\sum_{0<n<N-1} w_{n} \delta^{(N)}(i-l-n) \delta^{(N)}(j-k+n),
$$

其中 $w_{n}$ 为任意参数. 进而, 将 (8) 式代人 (1) 式有

$$
\begin{aligned}
\sum_{\lambda \mu \nu} \bar{S}_{\mu \nu}^{i j} \bar{S}_{\lambda n}^{j k} \bar{S}_{l m}^{\mu \lambda} \omega^{l+m+n-i-i-k} & -\sum_{\lambda \mu \nu} \bar{S}_{\mu \nu}^{i j} \bar{S}_{\lambda n}^{\nu k} \bar{S}_{l m}^{\mu \lambda} \omega^{(\lambda+r)+(\mu-r)+(\nu-\beta)-(\nu+\alpha)-(\mu-\alpha)-(2-\beta)} \\
& -\sum_{k \mu \nu} \bar{S}_{\mu \nu, j}^{i j} \bar{S}_{2 n}^{\nu k} \bar{S}_{l m}^{\mu \lambda},
\end{aligned}
$$

也即 (8) 式确实是 $Z_{N}$ 的 BGR 的解. 为了具体化, 我们给出 $N=3$ 时的形式, 其行列指 标安排为 $(i j)=11,12,21,13,22,31, \cdots, N N$ :

$$
S=\left[\begin{array}{cccccccccc}
w_{0} & & & & & & \omega^{2} w_{1} & \omega w_{2} & \\
& w_{2} & \omega^{2} w_{0} & & & & & & \omega w_{2} \\
& \omega w_{0} & w_{1} & & & & & & \omega^{2} w_{1} \\
& & & w_{1} & \omega^{2} w_{2} & \omega w_{0} & & & \\
& & & \omega w_{2} & w_{0} & \omega^{2} w_{1} & & & \\
& & & \omega^{2} w_{0} & \omega w_{1} & w_{2} & & & \\
\omega w_{1} & & & & & & w_{2} & \omega^{2} w_{0} & \\
\omega^{2} w_{2} & & & & & & \omega w_{0} & w_{1} & \\
& \omega^{2} w_{1} & \omega w_{2} & & & & & & \omega w_{0}
\end{array}\right]
$$

$\omega^{3}-1, w_{0}, w_{1}, w_{2}$ 为任意参数,式中空白项表示矩阵元为零.

当 (13) 式中取 $\omega-1$ 时即回到以前的结果 ${ }^{[4,5]}$. 观察 (8) 式, 它包含了参数 $\omega$, 即 1 的 $N$ 次方根. 实际上, 可以把它视为量子变形参数 $q$ 且 $q^{N}=1$, 进而可以讨论其代数结构.

\section{二、Weyl 代 数}

由 $\mathrm{F}-\mathrm{R}-\mathrm{T}^{[n]}$ 的基本精神, 给定 $S$, 利用 (5) 式即可决定出其相应代数的对易关系. 对通 常的 $q$ 变形情况, 它即给出量子代数的对易关系. 现在我们的问题是: 给定形如 (8) 式的 $S$, 求出它通过 F-R-T 决定出的算子关系. 由于 $x-\left(x_{i j}\right)$ 满足 coproduct

$$
\Delta\left(x_{i j}\right)-\sum_{k=1}^{N} x_{i k} \otimes x_{k j},
$$

我们将对 (8) 式给出的 $S$, 决定出其表示矩阵中矩阵元作为算子的具体组成.

以下我们将证时表示 


$$
\pi\left(x_{i j}\right)-w_{i-i} Z X^{i-i} \omega^{i-1}
$$

满足 (5) 式, 如果 $S$ 满足 (8) 式, $Z$ 与 $X$ 满足 Weyl 关系

$$
Z X-\omega X Z, \omega^{N}-1 \text {. }
$$

证因为 (5) 式可具体表为

$$
\sum_{k, l} S_{k l}^{i i}\left(x_{k m} x_{l n}\right)-\sum_{k, l}\left(x_{i k} x_{i l}\right) S_{m n}^{k l},
$$

将 (15) 式代入(17)式, 并利用 (8) 式得

$$
\begin{aligned}
& \sum_{\mu, k, l} w_{\mu} w_{m-K^{2}} w_{n-l} \delta^{(N)}(i-l-\mu) \delta^{(N)}(j-k+\mu) \omega^{i-k} Z X^{m-k} \omega^{k-1} Z X^{n-l} \omega^{l-1} \\
& \quad-\sum_{\mu, k, l} w_{\mu} w_{k-i} w_{l-j} \delta^{(N)}(k-n-\mu) \delta^{(N)}(l-m+\mu) \omega^{k-m} Z X^{k-i} \omega^{i-1} Z X^{l-i} \omega^{i-1},
\end{aligned}
$$

利用 Weyl 关系 (16) 式,经过计算, (18) 式可简化为

$$
\begin{aligned}
& \sum_{\mu, k, l} w_{\mu} w_{m-k} w_{n-l} \delta^{(N)}(i-l-\mu) \delta^{(N)}(j-k+\mu) \\
& -\sum_{\mu, k, l} w_{\mu} w_{k-i} w_{l-i} \delta^{(N)}(k-n-\mu) \delta^{(N)}(l-m+\mu),
\end{aligned}
$$

显然 (19) 式平凡满足. 因此我们断言，(15) 式给出了 Weyl 对易关系. 另一种表示为

$$
\pi^{\prime}\left(x_{i j}\right)-w_{i-i} Z X^{i-i} \omega^{-(i-1)},
$$

它实际就是 De Vega 所提出的构造 $\pi\left(x_{i j}\right)$ 的 $\mathrm{Z}_{\mathrm{N}}$ 实现 ${ }^{[8]}$ :

$$
\pi^{\prime}\left(x_{i i}\right)_{k l}=S_{i i}^{k i} \text {. }
$$

直接计算给出实际例子, 当 $N=3$, 有

$$
\pi^{\prime}(x)=\left[\begin{array}{ccc}
w_{0} Z & w_{1} Z X & w_{2} Z X^{2} \\
w_{2} \omega Z X^{2} & w_{0} \omega Z & w_{1} \omega Z X \\
w_{1} \omega^{2} Z X & w_{2} \omega^{2} Z X^{2} & w_{0} \omega^{2} Z
\end{array}\right] .
$$

将 (22) 式代人 (17) 式,应用 (8) 式,计算表明与 (16) 式一致.

事实上, Weyl 代数亦为多参数量子群 $G L_{p i i, q i}(N)$, 其中 $p_{i j}^{-1}-q_{i j}-\omega^{i-i}$.

\section{三、结 论}

我们已经建立起一种新型的 $Z_{N}$ 模型的 BGR, 并通过 F-R-T 证明了它对 应于 Weyl 代数. 在这个过程中参数 $\omega^{N}-1$ 作为量子群参数起了关键性的作用.

致谢: 作者感谢景乃桓同志, 文中许多结果实际上是我们共同研究的成果; 特别感谢杨振宁教授的鼓 励、帮助, 也感谢 M. Jimbo 的讨论.

参考文献

[1] Baxter, R. J., Exactly Solved Models in Statistical Mechanics, Academic Press, New York, 1982.

[ 2 ] Drinfeld, V. G., Proceedings of ICM, Berkeley, California, USA, 1986.

[3] Sklyanin, E. K., Funct. Anal. Appl, 16(1982), 27.

[ 4 ] Ge, M. L., Gwa, L. H. and Zhao, H. K., J. Phys., 23A (1990), $L 795$.

[5] Ge, M. L., Xue, K. and Zhao, H. K., Phys. Lett., 151A(199n), 145.

[6] Tracy, C., Physica, D16(1985), 203.

[ 7 ] Faddeev, L. D., Reshetikhin, N. Yu. and Takhtajan, L. A., Leningrad Math. J, 1(1990), 193.

[ 8 ] De Vega, H. J., Inter. J. Mod. Phys., B4(1990), 735. 